\title{
SYMPOSIUM D
}

\section{Scientific Basis For Nuclear Waste Management}

The Seventh International Symposium on the Scientific Basis for Nuclear Waste Management attracted 129 invited and contributed papers. This year's symposium differed from those of previous years in that the geomedia-specific research relating to repository placement was emphasized. The media-specific theme was timely since United States repository sites have recently taken on expanded roles for development of site-specific information relative to repository licensing and also because basic research activities in other countries are becoming more focused. The geomedia that have received the largest amount of research applicable to nuclear waste disposal are basalt, salt, tuff, and granite. A half-day session was devoted to materials research related to disposal of nuclear waste in each of the four media types. Interactive phenomena between containment materials, radionuclide release and radiation/radiolysis effects were among the research reported in the environments characteristic of each geomedia.

Some of the significant findings reported included the incorporation of low alloy steels into aqueous environments containing silicate waste forms in enhanced reaction rates and colloidal particle formation. The iron also serves as an effective scavenger for many radionuclides. Radiolysis effects were found to generally enhance radionuclide release rates and metallic barrier materials corrosion rates. Alpha radiolysis was particularly effective at creating an oxidizing environment in salt brine, which can have significant effects on actinide solubility limits. Ionizing radiation (gamma) was shown to cause metallic sodium generation in bedded rock salt. Additionally, models for predicting radionuclide release from repositories in each geomedia were described.

The four media-specific sessions were evaluated on a technical basis by three distinguished members of the scientific community not associated with any geomedium (repository site). The review panel members were Dr. G.A. Cowan, Los Alamos Scientific Laboratory; Dr. C.A. Heath, NUS Corporation, and Prof. D.D. Runnells, University of Colorado. Their review was frank and even-handed. All three review panel members agreed that nuclear wasterelated research has come a long way in the past several years and that the important factors are now being considered and evaluated. They all agreed that the quality of research has improved and that a much better level of understanding is evident. However, the geomedia-specific research was criticized for its lack of focus and for a lack of communication between scientists and managers in the various research areas. Concern was expressed that the requirements for licensing a repository (both NRC and EPA) are not being adequately considered in nuclear waste disposal-related research. The end product of this research is supposed to be to demonstrate the licensability of a

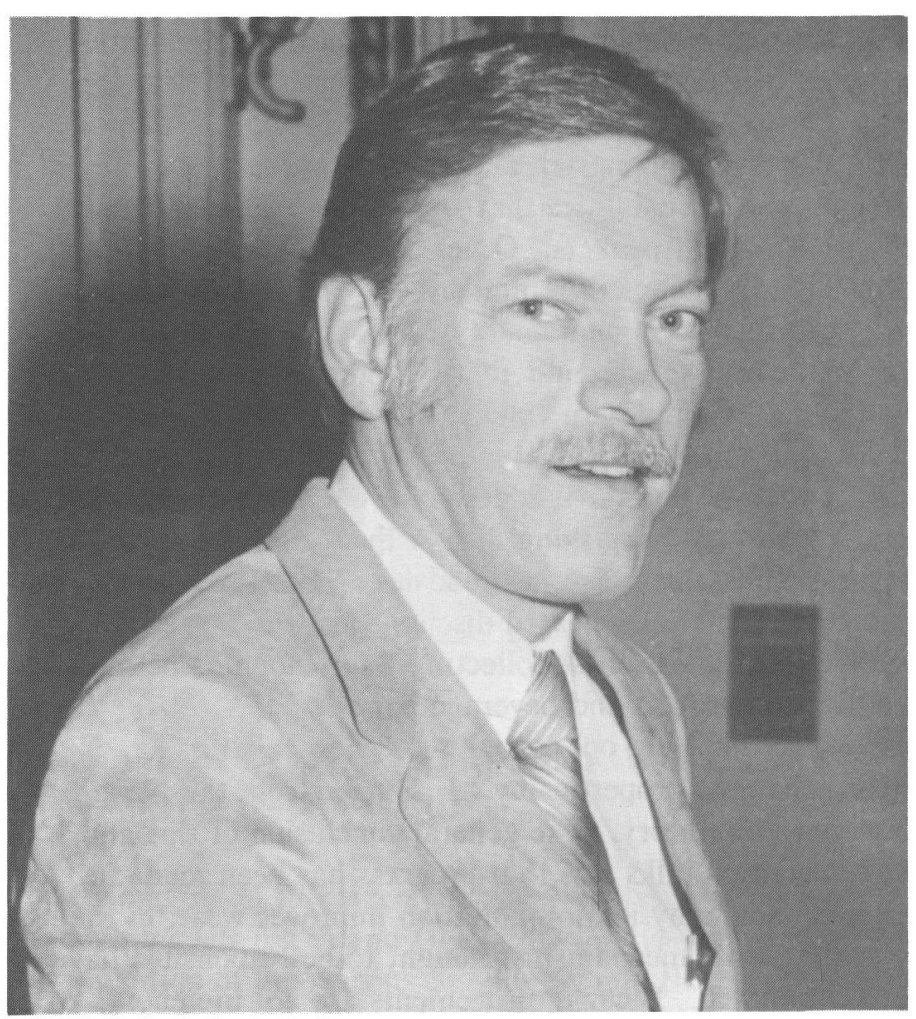

\section{G.L. MCVAY}

repository in a specific geomedium. Modeling activities of the entire repository system are critical and are not adequately addressed in existing programs. Model validation experiments and sufficient error analyses are missing from most geomedia-specific research activities. Materials testing conditions are often not relevant to realistic repository environments.

The audience reaction to the review panel comments varied from "right on" to rather mild disagreement.

However, it was clear that the review panel was a high point of the symposium and that a large amount of discussion and interest was generated from the audience.

In addition to the review panel, there were six invited speakers who covered key topics of high interest. These topics included the role of colloids in nuclear waste disposal, repository release modeling, solubility constraints, and the effects of microbes in ground water. An invited presentation topic of particular interest was a summary of the recent National Academy of Sciences report on radioactive waste management by Prof. T.H. Pigford of the University of California/Berkeley. Professor Pigford summarized a two- 


\section{SYMPOSIUM L}

\section{Plasma Processing And Synthesis Of Materials}

Symposium $\mathrm{L}$ was well attended, with the number of participants exceeding 150 .

The three-day program began with a set of plenary lectures providing a review of the current state of the art. The topics covered were:

"Overview of Plasma Processing" (J. Szekely)

"Plasma Generation" (E. Pfender)

"Plasma Diagnostics" (P. Fauchais)

"Modeling of Plasma Processes" (M. Boulos)

"Thermal Plasma Melting/Remelting Technology" (W. Roman)

"Plasma Extractive Metallurgy" (W.H. Gauvin)

"Rapid Solidification, Particle Deposition" (D. Apelian)

"Research Needs in Arc Technology" (J.V.R. Heberlein)

This overview was followed by four separate sessions, devoted to the following topics:

- Gas-Solid Reactions and Extractive Metallurgy in

Thermal Plasmas

- Plasma Synthesis, Melting and Coatings

- Properties of Materials Produced in Plasmas

- Plasma Diagnostics

Many of the papers provoked a lively discussion. The conference provided further evidence of the renewed interest in plasma processing and of the great need to build bridges between the research efforts aimed at processing on the one

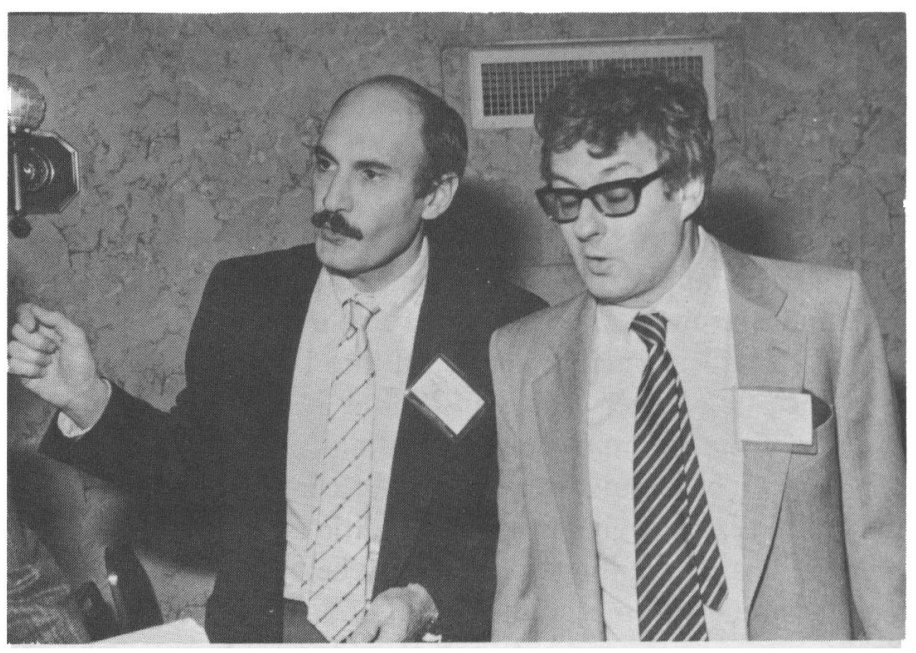

\section{J. APELIAN (left) and J. SZEKELY}

hand and on evaluating structure-property relationships on the other.

\section{Apelian}

Drexel University

J. Szekely

Massachusetts Institute of Technology

Chairmen

\section{SYMPOSIUM D}

\section{[Continued from Page 24]}

year investigation of the adequacy and relevance of nuclear waste-related research for repository licensing. One of the many conclusions presented was that current radionuclide release experiments are not conducted in a manner usable for repository licensing. This is because the nuclear waste form sits in an infinite volume of water in the repository environment and, under most conditions, the radionuclide release is determined at least partially by liquid phase diffusion. Experiments, on the other hand, are typically done in either a static environment with a fixed, rather small, volume of water or in a flowing environment where the flow rates are sufficiently high (because of experimental difficulties) so as to not allow any liquid phase diffusion control. Professor Pigford suggested dynamic experiments conducted in large vessels containing a porous medium.

A poster session was held on Wednesday evening from 7 to $10 \mathrm{p} . \mathrm{m}$. in conjunction with three other symposia. There were 31 posters for Symposium D and comparable numbers for the other symposia, which resulted in a large and rather well attended poster session. Food and drink served at the poster session added to its success.

Because of the large number of high quality papers submitted, parallel sessions were conducted on Thursday, the last day of the meeting. This was the first time that parallel sessions have been utilized for Symposium D. Both sessions were reasonably well attended, but the desirability of continuing double sessions in the future is questionable due to the competition between interesting papers.

\section{G.L. McVay}

Pacific Northwest Laboratory

Chairman

Symposium Support

Department of Energy

Nuclear Regulatory Commission 